\title{
Tratamiento de la imagen corporal en los trastornos de la conducta alimentaria desde la terapia online
}

\author{
Isabel Fernández Felipe \\ al260660@uji.es \\ Helio Marco Salvador \\ joseheliodoro.marco@ucv.es \\ Soledad Quero Castellano \\ squero@uji.es
}

\section{Resumen}

La insatisfacción corporal es uno de los factores predisponentes de los trastornos de conducta alimentaria (TCA) y las distorsiones perceptivas del tamaño corporal es un criterio diagnóstico de estos. Actualmente, se han desarrollado intervenciones online para reducir los factores de riesgo de los TCA. El objetivo de este trabajo es llevar a cabo una revisión de la literatura científica sobre el tratamiento de la imagen corporal en los TCA desde la terapia online y poner de manifiesto los avances generados. Se realizó una búsqueda en las bases de datos PsycNET, Scopus y Web of Science. La búsqueda se centró en revisar los programas de intervención para TCA con TIC en el tratamiento de la imagen corporal en TCA. Se encontraron 9 artículos cuyos resultados indican la existencia de diversos programas online como eBody Project, Student Bodies, Set Your Body Free y Healthy Body Image sobre imagen corporal. Los resultados indican, por un lado, que eBody Project y Student Bodies se asocian a una reducción significativa de síntomas de TCA y, por otro lado, Healthy Body Image y Set Your Body Free mostraron mejoras ligeramente significativas en el tratamiento de la imagen corporal para bulimia y trastorno por atracón. La investigación es escasa y presenta muchas limitaciones. Es necesario continuar investigando sobre programas de tratamiento online para la imagen corporal y los TCA ya que el uso de Internet cuenta con múltiples ventajas.

Palabras clave: trastornos alimentarios, tratamiento online, imagen corporal, bulimia nerviosa, anorexia nerviosa.

\section{Abstract}

Body dissatisfaction is one of the predisposing factors of eating disorders (ED) and perceptual distortions of body size are a diagnostic criterion of these. Currently, online interventions have been developed to reduce the risk factors of ED. The aim of the work is to carry out a review of the scientific literature on the 
treatment of body image in ED from online therapy and to show the progress made. A search was made in PsycNET, Scopus and Web of Science databases. The search focused on reviewing intervention programs for ED with ICTs for the treatment of the body image in ED. We found 9 articles whose results indicate the existence of several online programs such as eBody Project, Student Bodies, Set Your Body Free and Healthy Body Image about body image. The results indicate, on the one hand, that the eBody Project and the Student Bodies are associated with a reduction in symptoms of eating disorders and, on the other hand, Healthy Body Image and Set Your Body Free showed slightly significant improvements in the treatment of body image for bulimia and binge eating disorder. Research is scarce and has limitations. It is necessary to continue investigating online treatment programs for body image and ED since the use of the Internet has many advantages.

Keywords: eating disorders, online treatment, body image, bulimia nervosa, anorexia nervosa.

\section{Introducción}

Los trastornos de la conducta alimentaria y de la ingesta de alimentos se definen, según el DSM-5, como una alteración persistente en la alimentación o en el comportamiento relacionado con esta, que lleva a una alteración en el consumo o la absorción de los alimentos y que causa un deterioro significativo de la salud física o el funcionamiento psicosocial (American Psychiatric Association 2013). Además, los trastornos de la conducta alimentaria (TCA) se definen también como enfermedades psiquiátricas graves, marcadas por alteraciones en el comportamiento, las actitudes y la ingestión de alimentos, generalmente acompañadas de intensa preocupación con el peso o con la forma del cuerpo (American Psychiatric Association 2013). Dentro de la clasificación de estos trastornos, se incluyen la anorexia nerviosa, la bulimia nerviosa, el trastorno por atracón, el trastorno de la conducta alimentaria especificado y el trastorno de la conducta alimentaria no especificado, además de otros trastornos alimentarios en la infancia como pica, rumiación y trastorno de restricción/evitación de la alimentación.

En los TCA existe una etiología multicausal debido a la interacción de factores biológicos, psicológicos y sociales que inciden en la conducta del individuo y que afectan mayoritariamente a adolescentes y jóvenes del sexo femenino (Sánchez, Fornons, Aguilar, Vaque y Milà 2015). Actualmente, los TCA constituyen un importante problema de salud en las sociedades desarrolladas (Navarro 2016) y han alcanzado una gran relevancia psicosociosanitaria por su complejidad, su peculiaridad, su gravedad, su interés psicosocial, su falta de consciencia de la enfermedad, su prevalencia, su curso prolongado con tendencia a la cronicidad y sus dificultades de diagnóstico y tratamiento (Ministerio de Sanidad y Política Social 2009).

Las alteraciones de la imagen corporal (IC) son una de las características diagnósticas y clínicas de mayor importancia en los TCA (Garner 2002; Nye y Cash 2006; Stice 2002). Las alteraciones de la IC son uno de los factores de pronóstico más importantes de la bulimia nerviosa y en la anorexia nerviosa (Fairburn, Peveler, Jones, Hope y Doll 1993; Stice y Shaw 2002; Vocks, Legenbauer, Rüddel y Troje 2007). La insatisfacción con el cuerpo y las alteraciones de la IC se encuentran asociadas con la falta de control sobre la comida, hacer dietas restrictivas, pérdida de peso, miedo a engordar y la existencia de atracones y vómitos. Es más, la persistencia de la insatisfacción corporal después del tratamiento de los TCA es uno de los mayores predictores de recaída en los TCA (Cash y Hrabosky 2004; Shisslak y Crago 2001).

Estudios acerca de la eficacia de la intervención cara a cara con la terapia cognitivoconductual (TCC) para las alteraciones de la IC indicaron que, cuando se interviene en 
las alteraciones de la IC, no solo se consigue un cambio mayor que cuando no se interviene en la IC, sino que, además, se consigue un año antes. Así pues, el tratamiento de la IC potencia la eficacia y acelera el proceso de cambio de la TCC para los TCA (Marco, Perpiñà y Botella 2013).

Por otro lado, las tecnologías de la información y la comunicación (TIC) han incrementado su uso de manera considerable en los últimos años. La promoción y la utilización de estos nuevos modelos de tratamiento para los trastornos mentales conlleva el aumento de beneficios para las personas afectadas (Botella et al. 2007). Así, en las últimas décadas ha aumentado el uso de Internet para administrar tratamientos psicológicos y, en la actualidad, existe amplia evidencia que apoya el uso de tratamientos online para los trastornos emocionales (Andrews, Newby y Williams 2015), así como para otros problemas psicológicos como los problemas de sueño (Cheng y Dizon 2012) o adicciones (Riper et al. 2014). En este sentido, distintos estudios de metaanálisis arrojan tamaños del efecto comparables a los obtenidos con los tratamientos tradicionales cara a cara y mayores que en las condiciones control (Andersson, Cuijpers, Calbring, Riper y Hedman 2014; Andrews, Newby y Williams 2015). Por último, cabe señalar que estos tratamientos online presentan una serie de ventajas tales como la capacidad de personalizar los tratamientos y hacerlos interactivos, mayor accesibilidad, menor coste y flexibilidad (Kazdin y Blase 2011).

Por lo que se refiere específicamente al problema que nos ocupa, actualmente se han desarrollado intervenciones online para reducir los factores de riesgo de los TCA, entre ellos la imagen corporal, pero los resultados todavía son muy limitados y es necesario continuar investigando en programas de prevención e intervención online para la imagen corporal y los TCA. El objetivo de este trabajo es llevar a cabo una revisión de la literatura científica sobre el tratamiento de la imagen corporal en los TCA desde la terapia online y poner de manifiesto los avances generados.

\section{Método}

Para la realización de esta revisión, en primer lugar, se llevó a cabo una extensa búsqueda de la literatura en formato electrónico sin límite temporal usando las siguientes bases de datos: PsycNET, Scopus y Web of Science. Debido a que el tema principal era el tratamiento de la imagen corporal en TCA desde la terapia online, las palabras claves en la búsqueda del material fueron: «eating disorders», «body image», "anorexia nervosa», «bulimia nervosa», «binge eating», «internet-based treatment» y "online intervention». En dicha búsqueda se incluían estudios en los que los TCA y el tratamiento de la imagen corporal desde la terapia online fueran tratados explícitamente, así como estudios en los que en el contenido de la investigación incluía alguna parte referente a las dos cuestiones de interés. Para la selección de los estudios mediante los que posteriormente se realizó la revisión, se plantearon los siguientes criterios de selección: 1) la muestra refiere pacientes con alto riesgo de TCA, diagnosticados de TCA y población normal; 2) el tema principal es el tratamiento online de la imagen corporal en TCA; 3 ) el idioma de las publicaciones es en inglés y en español; y 4) el estudio es empírico. Se excluyeron las investigaciones realizadas por estudiantes así como los artículos repetidos en las diferentes bases de datos.

Todas las publicaciones que contenían de manera conjunta las palabras clave fueron incluidas $(n=12)$ y, a partir de ahí, los artículos fueron seleccionados en base a los criterios de inclusión y exclusión mencionados anteriormente. Para ello, se procedió a analizar, en primer lugar, si en el título y en el resumen se incluían los objetivos principales de la publicación. Posteriormente, se analizó la metodología y los resultados y, por último, el contenido del mismo. 
Como se puede observar en el diagrama de flujo presentado en la figura 1 , se encontraron un total de 12 artículos, de los cuales 3 fueron eliminados, obteniendo finalmente 9 artículos.

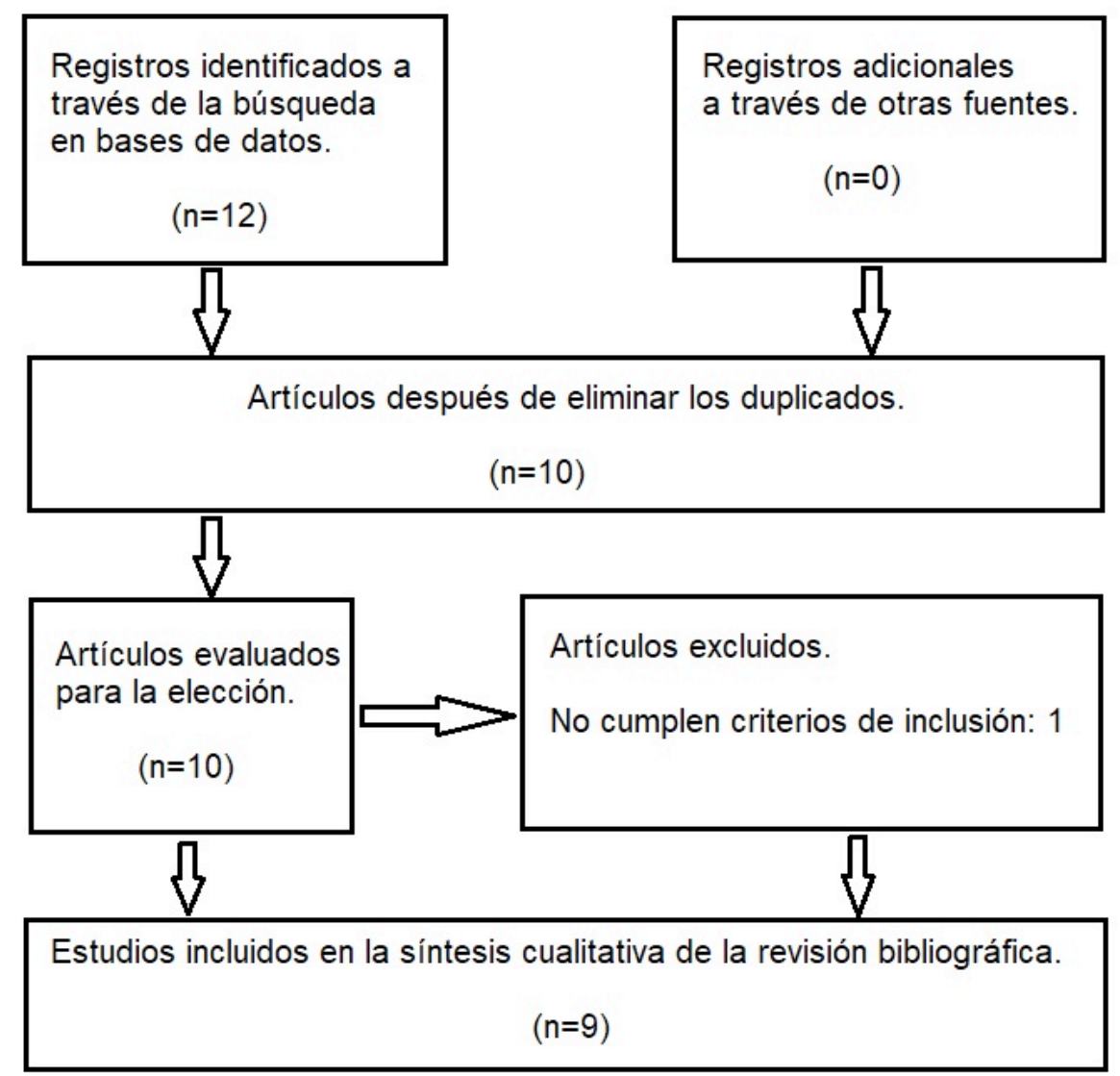

Figura 1. Diagrama de flujo ilustrando el proceso de búsqueda y la selección de la literatura.

\section{Resultados}

A continuación se describen los 9 estudios seleccionados dispuestos en función de la relevancia de los resultados obtenidos, siendo el último el de más importancia. Los resultados indican la existencia de cuatro programas online: Set Your Body Free, Healthy Body Image, eBody Project y Student Bodies.

El programa Set Your Body Free es un programa de intervención guiada autoaplicada cuyos componentes de intervención son la psicoeducación, el tratamiento de la imagen corporal, la solución de problemas y la motivación para el cambio. Se encontraron dos estudios acerca de este programa de tratamiento. El primero consideraba una muestra de 40 participantes diagnosticadas de bulimia nerviosa y trastorno por atracón (Gollings y Paxton 2006), mientras que el segundo incluía 73 participantes con sintomatología de TCA (Heinicke, Paxton, McLean y Wertheim 2007). Los resultados de este tratamiento indicaron que el tamaño del efecto desde la preintervención a la posintervención fue pequeño en comparación con otros programas de tratamiento online para TCA como eBody Project o Student Bodies.

Por otro lado, el programa Healthy Body Image es un programa guiado de autoayuda de promoción e intervención de la salud en TCA (excepto anorexia nerviosa de síndrome completo). Los componentes de este programa son el tratamiento en autoestima, actitudes y comportamientos alimentarios e imagen corporal. Se 
encontraron en tres estudios cuyas muestras fueron, en primer lugar, 1151 participantes divididos en tres grupos: «bajo riesgo», "alto riesgo» y "referencia clínica» (Jones et al. 2014). Estos últimos cumplían criterios de anorexia nerviosa, bulimia nerviosa y trastorno por atracón. El segundo estudio estaba compuesto por una muestra de 421 participantes sin sintomatología clínica (McCabe, Ricciardelli y Karantzas 2010) y el tercer estudio por 415 participantes sin sintomatología clínica (Jones y Crawford 2006). Los resultados de este programa mostraron mejoras ligeramente significativas en las actitudes relacionadas con la imagen corporal.

En tercer lugar, el eBody Project es un programa de intervención a través de un foro cuyos componentes engloban la confrontación al ideal de delgadez, la motivación para el cambio, el entrenamiento en habilidades, el apoyo social y la cohesión grupal para crear disonancia. En la búsqueda, se encontraron dos estudios. El primero constaba de una muestra de 107 participantes con alto riesgo de desarrollar un TCA (Stice, Durant, Rohde y Shaw 2014) y el segundo estudio refería una muestra de 680 participantes sin sintomatología clínica (Stice, Rhode, Durant y Shaw 2012). Los resultados de este programa mostraron reducciones significativas previas a los factores de riesgo y los síntomas de TCA respecto al grupo control. Además, los efectos no difirieron significativamente para eBody respecto a la intervención grupal y, finalmente, los resultados mostraron que este programa era tan eficaz como la intervención grupal.

Por último, el Student Bodies es un programa de intervención que incluye un módulo de psicoeducación y un grupo de noticias para la comunicación que consistían en lecturas semanales y publicaciones. Los componentes de este programa son el tratamiento de la imagen corporal, la educación sobre la nutrición, los trastornos de la conducta alimentaria, el ejercicio y la regulación de peso. Se encontraron dos estudios cuyas muestras fueron, por un lado, un estudio de 65 participantes con sintomatología subclínica de TCA (Saekow et al. 2015) y, por otro lado, 72 participantes con alto riesgo de desencadenar un TCA (Low et al. 2006). Los resultados indicaron que el programa redujo con éxito las preocupaciones sobre la imagen corporal y previno el desarrollo de los TCA.

\section{Discusión y conclusiones}

Los tratamientos de la imagen corporal en TCA desde la terapia online han sido estudiados por diversos autores. A lo largo de la realización de esta revisión bibliográfica hemos podido observar que tanto el tipo de estudio como los resultados son muy variados.

En los estudios utilizados para la revisión, se encuentran cuatro programas para el tratamiento de la imagen corporal en TCA desde la terapia online: Set Your Body Free, Healthy Body Image, eBody Project y Student Bodies. Estos cuatro programas de intervención se han desarrollado para el tratamiento de la imagen corporal desde la terapia online, así como para otros aspectos importantes en el tratamiento como son el patrón alimentario, la motivación, la psicoeducación, etc. Se han estudiado en diferentes tipos de muestras: pacientes con alto riesgo de desarrollar un TCA, pacientes diagnosticados de TCA y participantes sin sintomatología clínica de TCA. Además, estos programas difieren en la modalidad de intervención, desde programas de autoayuda guiado, programa a través de un foro e intervención guiada autoaplicada hasta un programa psicoeducativo y un grupo de noticias para la comunicación.

Los resultados nos muestran que, en las muestras con alto riesgo de desarrollar un TCA, el eBody Project y el Student Bodies se asocian a una reducción significativa de síntomas de TCA, entre ellos, la distorsión de la imagen corporal, de forma ligera. Por otro lado, en la muestra de pacientes diagnosticados de TCA, los resultados de eficacia de terapia online para la bulimia nerviosa y el trastorno por atracón no están 
claros y no se sabe nada para la anorexia nerviosa, tal y como se observa en los programas de Healthy Body Image y Set Your Body Free.

De lo anterior, podemos incluir que la investigación en este ámbito de estudio es escasa y presenta limitaciones. Una primera limitación que hay que destacar es que la muestra en algunos estudios es pequeña y, por otra parte, no todos los estudios incluyeron población clínica.

Por otra parte, también cabe señalar que las revisiones bibliográficas tienen limitaciones caracterizadas por una serie de sesgos. Uno de ellos es el sesgo de publicación. Deriva de que muchos trabajos científicos, la mayoría con resultados que no hallan diferencias significativas o resultados en contra de la hipótesis de estudio, nunca llegan a publicarse, tardan más tiempo en hacerlo o se citan menos. Todo esto condiciona los resultados y puede dar lugar a sesgos en la revisión. También contribuye a este tipo de sesgo la duplicación de estudios o ignorar los trabajos publicados en idiomas diferentes a los referidos en los criterios de inclusión. Junto con este sesgo, también aparece el sesgo de selección, el cual deriva de la definición clara de los criterios de inclusión y exclusión y la objetividad de estos. Pueden sesgarse los resultados ya que estos dos criterios pueden verse influenciados por los resultados del mismo. Además, otros aspectos que pueden comprometer la validez de los resultados de una revisión son la calidad de los estudios incluidos, la variabilidad entre estos o los errores en la fase de análisis. Y, en tercer lugar, las limitaciones acerca de la disponibilidad de los textos completos de algunas publicaciones importantes, que no parecen dispuestas por ahora a ceder gratuitamente sus contenidos y que pueden ser muy relevantes y novedosas en nuestro estudio.

Además, la muestra en algunos de estos estudios sigue siendo limitada ya que no todos referían población clínica.

En lo que concierne a líneas de investigación futuras, en primer lugar, sería interesante incluir muestras clínicas en las investigaciones futuras ya que, como se ha mencionado anteriormente, muchos de estos estudios no la incluían. Esto puede haber llevado a la obtención de resultados diferentes en relación a la eficacia y en la comparación de programas que no trabajan con población clínica o con población de alto riesgo. En este sentido, creemos que es necesario llevar a cabo más estudios con pacientes diagnosticados de TCA con la finalidad de diseñar un protocolo de tratamiento online basado en la evidencia para la imagen corporal. En segundo lugar, también son necesarios más estudios que incorporen intervenciones online dirigidas a corregir alteraciones de la imagen corporal, pero aplicadas a poblaciones en riesgo con una finalidad preventiva.

En suma, la terapia online en el campo de los TCA requiere más atención debido a sus múltiples ventajas ya señaladas, como la capacidad de personalizar las intervenciones y hacerlas interactivas, mayor acceso, menor coste y flexibilidad, entre otros, ya que, en la actualidad, estos programas son básicos en forma y contenido y utilizan poco estas grandes ventajas (Kazdin y Blase 2011).

\section{Referencias bibliográficas}

American Psychiatric Association. 2013. Diagnostic and Statical Manual of Mental Disorders (5th ed.). Washington, DC: American Psychiatric Association.

Andersson, Gerhard, Pim Cuijpers, Per Carlbring, Heleen Riper y Erik Hedman. 2014. «Guided Internet-based vs. face-to-face cognitive behavior therapy for psychiatric and somatic disorders: a systematic review and meta-analysis». World Psychiatry 13(3): 288-295. 
Andrews, Gavin, Jill M. Newby y Alishia D. Williams. 2015. "Internet-delivered cognitive behavior therapy for anxiety disorders is here to stay». Current Psychiatry Reports 17(1): 533-533.

Botella, Cristina, Rosa María Baños, Azucena García-Palacios, Soledad Quero, Verónica Guillén y Heliodoro Marco. 2007. «La utilización de las nuevas tecnologías de la información y la comunicación en psicología clínica». Uoc Papers 4: 32-41.

Cash, Thomas y Joshua I. Hrabosky. 2004. «Treatment of body image disturbances». En Handbook of eating disorders and obesity, ed. J. Kevin Thompson (515541). Hoboken, NJ: Wiley.

Cheng, Sammy K. y Janine Dizon. 2012. «Computerised cognitive behavioural therapy for insomnia: a systematic review and meta-analysis». Psychotherapy and Psychosomatics 81(4): 206-216.

Fairburn, Christopher G., Robert C. Peveler, Rosemary Jones, Ronald Anthony Hope y Hellen Doll. 1993. «Predictors of 12-month outcome in bulimia nervosa and the influence of attitudes to shape and weight». Journal of Consulting and Clinical Psychology 61: 696-698.

Garner, David M. 2002. "Body image and anorexia nerviosa». En Body image: A handbook of theory, research, and clinical practice, ed. Thomas Cash y Thomas Pruzinsky (295-303). New York: Guilford.

Gollings, Emma K. y Susan Paxton. 2006. «Comparison of Internet a face-to-face delivery of a group body image and disordered eating intervention for women: a pilot study». Eating disorders 14(1): 1-15.

Heinicke, Brooke E., Susan J. Paxton, Siân McLean y Eleanor Wertheim. 2007. "Internet-delivered targeted group intervention for body dissatisfaction and disordered eating in adolescent girls: a randomized controlled trial». Journal of Abnormal Child Psychology 35(3): 379-391.

Jones, Diane Carlson y Joy K. Crawford. 2006. "The peer appearance culture during adolescence: Gender and body made variations». Journal of Youth and Adolescence 2: 257-269.

Jones, Megan, Andrea E. Kass, Mickey Trockel, Alan Glass, Denise E. Wilfley y C. Barr Taylor. 2014. «A population-wide screening and tailored intervention platform for eating disorders on college campuses: the Healthy Body Image program». Journal of American College Health 62(5): 351-356.

Kazdin, Alan E. y Stacey L. Blase. 2011. "Rebooting psychotherapy research and practice to reduce the burden of mental illness». Perspectives on Psychological Sciences 6(1): 21-37.

Low, Kathryn Graff, Swita Charanasomboon, Jill Lesser, Katie Reinhalter, Rachel Martin, Hannah Jones, Andy Winzelberg, Liana Abascal y C. Barr Taylor. 2006. «Effectiveness of a Computer-Based Interactive Eating Disorders Prevention Program at Long-Term Follow-Up». Eating Disorders 14(1):17-30.

Marco, José H., Conxa Perpiñá y Cristina Botella. 2013. «Effectiveness of cognitive behavioral therapy supported by virtual reality in the treatment of body image in eating disorders: one year follow-up». Psychiatry Research 209(3): 619-625.

McCabe, Marita, Lina A. Ricciardelli y Gery C. Karantzas. 2010. «Impact of a Healthy Body Image program among adolescent boys on body image, negative affect and body changes strategies». Body Image 7(2): 117-123.

Ministerio de Sanidad y Política Social. 2009. Guía de Práctica Clínica sobre la Prevención y el Tratamiento de la Obesidad Infanto-Juvenil. Comunidad de Madrid: Agencia de Calidad del Sistema Nacional de Salud.

Navarro, María del Mar 2016. «Prevención de los trastornos de la conducta alimentaria en adolescentes: Una propuesta de intervención en Institutos de Educación Secundaria». Trabajo de Fin de Máster. Barcelona: Universitat Oberta de Catalunya.

Nye, Stacey y Thomas F. Cash. 2006. «Outcome of manualized cognitive-behavioral body image therapy with eating disordered women treated in a private clinical practice». Eating disorders 14: 31-40. 
Riper, Heleen, Matthijs Blankers, Hana Hadiwijaya, John A. Cunningham, Stella Clarke, Reinout W. Wiers, David Daniel Ebert y Pim Cuijpers. 2014. «Effectiveness of Guided and Unguided Low-Intensity Internet Interventions for Adult Alcohol Misuse: A Meta-Analysis». PLoS ONE 9(6): 1-11.

Saekow, Jenine, Megan Jones, Elise Gibbs, Corinna Jacobi, Ellen E. FitzsimmonsCraft, Denise E. Wilfley y C. Barr Taylor. 2015. «StudentBodies-eating disorders: A randomized controlled trial of a coached online intervention for subclinical eating disorders». Internet Interventions 2: 419-428.

Sánchez, Violeida, David Fornons, Alicia Aguilar, Cristina Vaque y Raimon Milà. 2015. «Evolución de los conocimientos acerca de los trastornos del comportamiento alimentarios, a través de una intervención educativa en estudiantes de enseñanza postobligatoria». Nutrición Clínica y Dietética Hospitalaria 35(1): 3241.

Shisslak, Catherine M. y Marjorie Crago. 2001. «Risk and protective factors in the development of eating disorders». En Body image, eating disorders, and obesity in youth: Assessment, prevention, and treatment, ed. J. Kevin Thompson y Linda Smolak (103-125). Washington, DC: American Psychological Asso ciation.

Stice, Eric. 2002. "Body image and bulimia nervosa». En Body image: A handbook of theory, research, and clinical practice, ed. Thomas F. Cash y Thomas Pruzinsky (304-311). New York: Guilford.

Stice, Eric y Heather Shaw. 2002. "Role of Body Dissatisfaction in the Onset and Maintenance of Eating Pathology: A Synthesis of Research Findings». Journal of Psychosomatic Research 53: 985-993.

Stice, Eric, Paul Rohde, Shelley Durant y Heather Shaw. 2012. «A preliminary trial of prototype Internet dissonance-based eating disorder prevention program for young women with body image concerns». Journal of Consulting and Clinical Psychology 80(5): 907-916.

Stice, Eric, Shelley Durant, Paul Rohde y Heather Shaw. 2014. «Effects of a prototype Internet dissonance-based eating disorder prevention program at 1- and 2-year follow-up». Health Psychology 33(12): 1558-1567.

Vocks, Silja, Tanja Legenbauer, Heinz Rüddel y Nikolaus Troje. 2007. «Static and Dynamic Body Image in Bulimia Nervosa: Mental Representation of Body Dimensions and Biological Motion Patterns». International Journal of Eating Disorders 40(1): 59-66. 